Published in final edited form as:

J Clin Oncol. 2005 October 1; 23(28): 7005-7012.

\title{
Postoperative Nomogram Predicting the 10-Year Probability of Prostate Cancer Recurrence After Radical Prostatectomy
}

\author{
Andrew J. Stephenson, Peter T. Scardino, James A. Eastham, Fernando J. Bianco Jr, Zohar \\ A. Dotan, Christopher J. DiBlasio, Alwyn Reuther, Eric A. Klein, and Michael W. Kattan \\ From the Department of Urology, Sidney Kimmel Center for Prostate and Urologic Cancers, \\ Memorial Sloan-Kettering Cancer Center, New York, NY; Glickman Urological Institute, and the \\ Department of Quantitative Health Sciences, Cleveland Clinic Foundation, Cleveland, $\mathrm{OH}$.
}

\begin{abstract}
Purpose- A postoperative nomogram for prostate cancer recurrence after radical prostatectomy (RP) has been independently validated as accurate and discriminating. We have updated the nomogram by extending the predictions to 10 years after RP and have enabled the nomogram predictions to be adjusted for the disease-free interval that a patient has maintained after RP.

Methods-Cox regression analysis was used to model the clinical information for 1,881 patients who underwent RP for clinically-localized prostate cancer by two high-volume surgeons. The model was externally validated separately on two independent cohorts of 1,782 patients and 1,357 patients, respectively. Disease progression was defined as a rising prostate-specific antigen (PSA) level, clinical progression, radiotherapy more than 12 months postoperatively, or initiation of systemic therapy.
\end{abstract}

Results - The 10-year progression-free probability for the modeling set was 79\% (95\% CI, 75\% to $82 \%)$. Significant variables in the multivariable model included PSA $(P=.002)$, primary $(P<$. $0001)$ and secondary Gleason grade $(P=.0006)$, extracapsular extension $(P<.0001)$, positive surgical margins $(P=.028)$, seminal vesicle invasion $(P<.0001)$, lymph node involvement $(P=$. $030)$, treatment year $(P=.008)$, and adjuvant radiotherapy $(P=.046)$. The concordance index of the nomogram when applied to the independent validation sets was 0.81 and 0.79 .

Conclusion-We have developed and validated as a robust predictive model an enhanced postoperative nomogram for prostate cancer recurrence after RP. Unique to predictive models, the nomogram predictions can be adjusted for the disease-free interval that a patient has achieved after RP.

\section{INTRODUCTION}

Accurate predictive models for prostate cancer recurrence after radical prostatectomy (RP) are essential for the rational application of adjuvant therapy and patient counseling; they may also be used to modify surveillance intervals and diagnostic tests on the basis of the individual patient's risk of recurrence. In clinical trials, accurate predictive models are essential to identify appropriate candidates for investigational therapy and to ensure comparability of treatment and control groups.

Numerous clinical and pathologic variables associated with disease recurrence after RP have been identified, including serum prostate-specific antigen (PSA), Gleason grade, pathologic

Address reprint requests to Michael W. Kattan, PhD, Chair, Department of Quantitative Health Sciences, The Cleveland Clinic Foundation, Wb4, 9500 Euclid Ave, Cleveland, OH 44195; e-mail: kattanm@ccf.org..

Presented in part at the 2004 Annual Meeting of the American Urological Association May 8-13, 2004, San Francisco, CA. 
stage, and surgical margins. In 1999, we developed a continuous multivariable postoperative nomogram on the basis of these variables that calculates a man's 7-year progression-free probability (PFP) after RP. ${ }^{1}$ The nomogram was developed on a single-surgeon cohort of 996 patients treated by RP alone at Baylor College of Medicine (BCM) between 1983 and 1997 and was internally validated with a bootstrap-corrected concordance index ( $c$-index) of 0.88 . We have, subsequently, demonstrated the nomogram to be accurate and discriminating in two separate independent validation studies. 2,3

Despite the robust information contained within the nomogram, it has certain limitations. While the 7-year end point for disease progression is a reasonable estimate of the likelihood of cure, it is known that clinically significant prostate cancer recurrences can occur after 7 years. ${ }^{4,5}$ In addition, in developing the nomogram, patients receiving adjuvant radiotherapy were considered as treatment failures. Thus, the predictions of the nomogram may be overly pessimistic, given that several of these patients would not have recurred without adjuvant therapy. Lastly, widespread PSA screening has resulted in a stage migration. Patients more recently diagnosed may have a more favorable prognosis after adjustment for other parameters. 6 The nomogram was developed on patients treated over a 14-year period beginning in 1984 (many of whom were treated before the introduction of the PSA assay), so the predictions may not be accurate for patients treated contemporaneously.

We have developed a new postoperative nomogram to address the limitations of the original model. The predictions of the nomogram have been extended to 10 years after RP, and the variables in the nomogram have been adjusted for the use of adjuvant radiotherapy and the year of treatment. Given that a patient's prognosis improves with the disease-free interval maintained after RP, we have also enabled the 10-year PFP to be adjusted to reflect this changing prognosis.

\section{METHODS}

\section{Patients}

Between 1983 and 2003, 4,158 patients with clinically-localized prostate cancer underwent RP without neoadjuvant therapy at Memorial Sloan-Kettering Cancer Center (MSKCC) and/ or by a single surgeon at BCM. For nomogram development, 2,043 patients treated by two high-volume surgeons at MSKCC or BCM were used as a modeling cohort. An additional 2,093 patients treated by other surgeons at MSKCC were set aside for independent validation. Thirty-two patients who had RP aborted for lymph node metastases identified by frozen-section analysis and 475 patients with missing values for the predictor variables were excluded from the analysis. This left 1,881 patients with complete records for nomogram development and 1,782 patients for independent validation (MSKCC validation set). A second validation cohort contained 1,357 patients treated by RP alone at the Cleveland Clinic Foundation (CCF) between 1990 and 2000 (CCF validation set). The clinical features of the modeling and validation cohorts are listed in Table 1.

All prostates were totally embedded and those resected from patients in the modeling cohort were sectioned by the whole-mount technique. Given that the prostatectomy specimens were not assessed by whole-mount sectioning in the MSKCC validation cohort, there may be a bias in the assessment of pathologic variables between the modeling and validation cohorts.

In general, patients were followed for disease recurrence postoperatively with serum PSA level determinations and clinical assessment every 3 months for the first 3 years, every 6 months in years 4 and 5, and annually thereafter. Diagnostic imaging modalities and/or anastomotic biopsy were reserved for patients who had evidence of biochemical recurrence. 


\section{Disease Progression}

Disease progression after RP was defined as a serum PSA value of $0.4 \mathrm{ng} / \mathrm{mL}$ or greater that was confirmed by a second measurement that was higher than the first by any amount, biopsyconfirmed local recurrence, distant metastases, cancer-specific mortality, or initiation of systemic therapy. Patients who received postoperative radiotherapy within 12 months of RP at PSA levels less than $0.2 \mathrm{ng} / \mathrm{mL}$ were considered as receiving adjuvant radiotherapy. Any patient who received radiotherapy more than 12 months postoperatively and/or for PSA values of $0.2 \mathrm{ng} / \mathrm{mL}$ or more (without meeting the criteria for biochemical recurrence) was considered a treatment failure.

\section{Statistical Analysis}

Estimates of the probability of remaining free of progression were calculated by the KaplanMeier method. ${ }^{7}$ Patients were censored if they were lost to follow-up or died from causes other than prostate cancer. Multivariable analysis was performed with Cox proportional hazards regression. Providing predictions on the basis of the disease-free interval was accomplished by the conditional survival approach. ${ }^{8}$ Because PSA had a skewed distribution and suspected nonlinear effect, it was modeled as a restricted cubic spline of its log-transformed value. ${ }^{9}$ Given that only 190 patients in the modeling cohort had had primary or secondary Gleason grades of 1,2 , and 5, Gleason grades were compressed into two categories (Gleason $\leq 3$ and $\geq 4$ ) for modeling purposes. As a result of insufficient data in the validation cohorts on the level of extracapsular extension (ECE), it was modeled as a binary category (Yes: focal or established ECE; No: capsular invasion or disease confined to the prostate stroma). ${ }^{10}$ Adjuvant radiotherapy was modeled as a time-dependent covariate. ${ }^{11}$ All decisions with respect to the coding of the variables were made before modeling.

For model validation, we assessed both discrimination and calibration. Discrimination refers to the ability of the nomogram to rank patients by their risk, such that patients with a higher predicted risk of treatment failure should be more likely to recur. Discrimination is easily quantifiable using the $c$-index, which is similar to an area under the receiver operating characteristic curve, and is applicable to time-to-event data. ${ }^{12}$ Calibration refers to the accuracy of the nomogram as measured by a visual inspection of the plots of predicted probability of progression versus actual outcome.

For internal validation, a jackknife (ie, leave-one-out) cross-validation procedure was used to develop 1,881 unique models, leaving one sample out at each iteration. The probability of disease recurrence was calculated for each sample withheld, and the $c$-index was calculated based on these predictions. Bootstrapping was not performed because of the difficulties encountered when time-dependent variables were incorporated into the model. The second component of internal validation was to compare the jackknife-corrected predicted probability of progression with the actual outcome (ie, calibration). For independent validation, the predicted probability of progression calculated by the nomogram was compared with the actual outcome of patients in the validation sets to generate a $c$-index and calibration plot. Because primary Gleason grade was unavailable for 310 patients with Gleason 7 disease in the CCF validation set, the validation was performed twice, first assuming these patients had Gleason $3+4$ cancer, then assuming they had Gleason $4+3$ cancer. Patients receiving adjuvant radiotherapy in the validation sets were censored at the time of radiotherapy. All statistical analysis was performed using $S$-Plus software (S-plus 2000; Insightful Corp, Redmond, WA) with additional functions (called "Design") added. All $P$ values resulted from use of two-sided statistical tests. The study was conducted under Health Insurance Portability and Accountability Act guidelines and received institutional review board approval from all participating institutions. 


\section{RESULTS}

In the modeling set, 183 progression events were observed, and the 10-year PFP was 79\% (95\% CI, 75 to 82; Fig 1). In the MSKCC and CCF validation sets respectively, 221 and 271 progression events were observed, and the 10-year PFP was 70\% (95\% CI, 64 to 75) and 67\% ( $95 \%$ CI, 63 to 71). For patients who were free of recurrence, the median follow-up was 25 months (range, 1 to 193) in the modeling set and 40 months (range, 1 to 134) and 53 months (range, 1 to 164) in the MSKCC and CCF validation sets, respectively.

Considering all 4,158 patients in the MSKCC-BCM cohort, the risk of subsequent disease progression for patients free of disease at 7 years and 10 years (PSA value less than $0.2 \mathrm{ng} / \mathrm{mL}$ at most 6 months before the 7- or 10-year mark or at any time afterward) was estimated. For the 1,212 patients who were free of disease at 7 years, 18 progression events were observed, and the actuarial probability of disease recurrence over the subsequent 8 years was $9 \%$ (95\% CI, 4 to 15). For the 284 patients who were free of disease at 10 years, five progression events were observed, and the actuarial probability of recurrence over the subsequent 5 years was $3 \%$ (95\% CI, $0 \%$ to $6 \%$ ).

Preoperative PSA $(P<.002)$, primary $(P<.0001)$ and secondary Gleason grades $(P=.0006)$, ECE $(P<.0001)$, positive surgical margins $(P=.028)$, seminal vesicle invasion $(P<.0001)$, lymph node involvement $(P=.030)$, treatment year $(P=.008)$, and absence of adjuvant radiotherapy $(P=.046)$ were all associated with disease progression in the multivariable model. A nomogram incorporating each of these clinical variables is shown in Figure 2. Note that the use of adjuvant radiotherapy is not included in the nomogram, as the model estimates the probability of disease recurrence after RP alone.

The graphical representation of the nomogram contains two components. The first step is to locate a patient's position on each predictor variable scale (surgical margins through PSA). Each scale position has corresponding prognostic points (bottom scale). The point values for all the predictor variables are summed to arrive at a total points value. The position of the total points value is then located on the $x$ axis of the graph above the variable scales, and a line is drawn vertically up from this point. The number of months that a patient has remained free of recurrence after RP (time zero being the immediate postoperative period) is then located on the $y$ axis of the graph, and a horizontal line is drawn from this point. On this graph, the slanted vertical lines ( 0.01 to 0.99 ) correspond to the 10 -year PFP calculated by the nomogram. The slanted vertical line nearest the intersection point of the horizontal line drawn from the time axis and the vertical line drawn from the total points axis indicates the 10-year PFP from the time of RP adjusted for the disease-free interval.

To demonstrate how to use the nomogram, a hypothetical patient who underwent RP in 2004 and had PSA 8.2, Gleason sum $7(3+4)$, ECE, positive margins, no seminal vesical invasion or lymph node involvement, and without adjuvant radiotherapy is calculated to have 134 total points. Immediately after RP, the intersection point of the vertical line drawn from 134 on the total points axis and the horizontal line drawn from time zero is nearest to the 0.8 slanted vertical line $(80 \% ; 10$-year PFP). If the same patient subsequently maintained an undetectable PSA for 60 months after RP, the intersection point of the vertical line drawn from 134 on the total points axis and the horizontal line drawn from 60 on the time axis is nearest the 0.9 slanted vertical line. The patient's likelihood of remaining free of progression for 10 years after RP improved from $80 \%$ to $91 \%$ by maintaining an undetectable PSA for 60 months.

The jackknife-derived $c$-index of the model was 0.86 . Figure 3 illustrates how the jackknifederived predictions of the nomogram compare with the actual outcome for the 1,881 patients. The 45 degrees line represents the performance of a perfectly calibrated model, in which 
predicted and actual outcomes perfectly correspond. The jackknife-derived predictions (solid line) closely approximate the actual outcome (45-degree line), indicating good calibration.

For independent validation, the 10-year PFP calculated from time zero after RP was applied separately to the MSKCC and CCF validation sets. The $c$-index of the nomogram in the MSKCC validation set was 0.81 . In the CCF validation set, the $c$-index of the nomogram was 0.78 assuming Gleason 7 patients were Gleason $3+4$, and 0.77 assuming Gleason 7 patients were Gleason $4+3$. Figure 4 illustrates the calibration plots of the nomogram when applied to patients in the MSKCC and CCF validation sets. The calibration plots suggest that the predictions of the nomogram may be too optimistic for patients with calculated 10-year PFP less than $90 \%$.

\section{DISCUSSION}

In 1999, we developed a continuous multivariable postoperative nomogram for prostate cancer recurrence after RP, and this model was subsequently validated to be an accurate and discriminating outcome prediction tool. ${ }^{1-3}$ Although the nomogram is considerably more complicated to use than risk-grouping schemata, the added complexity of nomograms results in enhanced predictive accuracy. Furthermore, the nomogram predictions are tailored to the risk posed by the characteristics of a individual's cancer, which is more relevant to the patient than group-level probabilities. ${ }^{13}$ The development of software for personal digital assistants and personal computers has facilitated their use. The software is freely available to the public (http://www.nomograms.org). The prostate cancer nomogram is currently the most widely used disease-specific prediction tool in oncology. ${ }^{14}$ Despite its popularity and validated accuracy, the postoperative nomogram has several limitations. The 7-year time period is not a definitive end point for estimating biochemical recurrence. Furthermore, the nomogram was developed and validated on patients treated in the 1980s and 1990s and does not account for the potentially improved prognosis of patients treated more contemporaneously.

To address these limitations, we have updated the postoperative nomogram by extending the predictions out to 10 years after RP. We have also enhanced the current version of the postoperative nomogram, as predictions are adjusted for patients' treatment year and for the use of adjuvant radiotherapy (which was considered as a treatment failure in the original model). To account for the changing prognosis of patients who have maintained an undetectable PSA for specific time intervals after RP, we have enabled the 10-year PFP prediction of the nomogram to be re-estimated based on the disease-free interval patients have achieved. The new postoperative nomogram is a robust predictive model with a calculated $c$-index of 0.81 and 0.78 when applied to separate independent validation sets.

The 10-year PFP would appear to be a sufficient end point for estimating the likelihood that a man will be cured of his prostate cancer by RP alone. Although the majority of recurrences are observed in the first 3 years after RP, ${ }^{15}$ a considerable number of patients may recur after 7 years and several of these may progress to bony metastases during their lifetime. ${ }^{4,5}$ However, of the 284 patients who were free of recurrence at 10 years after RP, only three progression events were observed and the estimated risk of subsequent progression over the following 5 years was $3 \%$.

Approximately half of the patients with a rising PSA level after RP will develop clinical recurrence during their lifetime and die of prostate cancer. ${ }^{4,16}$ Although biochemical progression may not be a clinically significant end point for all patients, it remains a valid end point to assess the efficacy of RP for cancer control. No patient would select, nor would any clinician recommend, an aggressive therapy that is destined to lead to biochemical progression, despite the potentially loose association with bone metastases. The majority of patients with 
biochemical recurrence will ultimately receive secondary hormonal or radiation therapy, both of which may be associated with significant morbidity. Of the 183 patients in the modeling cohort who experienced disease progression, the 5-year actuarial probability of remaining free of distant metastasis and/or secondary therapy from the time of biochemical recurrence was $16 \%$ (95\% CI, 10 to 21). Patients experiencing biochemical recurrence may also have a reduced health-related quality of life, even in the absence of disease-related symptoms. ${ }^{17}$

It is known that the risk of prostate cancer recurrence declines sharply beyond the first 2 years after RP, and recurrences after 6 years are uncommon. ${ }^{15}$ A feature of the new version of the postoperative nomogram is the ability to recalculate a patient's 10-year PFP after RP on the basis of the disease-free interval achieved. To our knowledge, the ability to re-estimate a patient's prognosis on the basis of subsequent events (in this case, maintaining an undetectable PSA for a specified period of time after RP) is rare in prediction models in medicine. The added feature of the nomogram will be useful for patient counseling and for the timing of surveillance clinical assessments and diagnostic tests.

The prognostic significance of treatment year in the new version of the nomogram is an intriguing finding. A possible explanation for the improved prognosis of contemporary patients may be related to stage migration as a significant reduction in median tumor volume has been observed among RP patients at our institution over the last decade (unpublished data). The fact that the presence of ECE, rather than the level of ECE, was used in the nomogram may partly explain the improved prognosis over time as proportionately more patients may have had focal rather than established ECE in recent years. Of note, the absence of the level of ECE in the new version of the nomogram is not indicative of its lack of prognostic significance for disease progression. The nomogram also does not take into account that the extent of lymph nodes involvement, seminal vesicle invasion, and positive surgical margins may have varied over time. As a result of widespread PSA screening and extended biopsy strategies, Stamey et al have argued that elevated PSA levels in contemporary patients is more reflective of the volume of benign prostatic hyperplasia rather than prostate cancer volume. ${ }^{18}$ Thus, the prognostic significance of elevated PSA levels in contemporary patients may be overestimated. Improvements in surgical technique over time may have contributed to improved outcomes after RP independent of the impact on surgical margins. The improved prognosis of treatment year may also be caused by a biologic phenomenon secondary to lead-time bias. Cancers detected early from aggressive diagnostic strategies may not have acquired the biologic properties of local invasion and metastasis compared to tumors of similar grade and pathologic stage that are further along in their natural history.

In the original nomogram, the use of adjuvant radio-therapy was considered a treatment failure despite the fact that many of these patients may not have progressed in the absence of adjuvant therapy. In the current version of the nomogram, the predictions were adjusted for adjuvant radiotherapy, which was included as a variable in the final model. Interestingly, adjuvant radiotherapy was a significant protective variable for disease progression after RP. The efficacy of adjuvant radiotherapy for preventing biochemical progression after RP was recently illustrated in a randomized phase III trial conducted by the European Organization for Research and Treatment of Cancer. ${ }^{19}$ The impact of adjuvant radiotherapy on metastatic progression is currently unknown.

Besides using biochemical progression as an end point, the nomogram has other limitations. Although the nomogram discriminated well among patients in the validation sets ( $c$-index 0.81 and 0.78 ), the probability of freedom from recurrence calculated by the nomogram tended to be overly optimistic. The apparent optimism of the nomogram may be explained by the different ways in which pathologic variables were assessed in the modeling and validation cohorts. The optimism of the nomogram may also be attributed to the fact that it was modeled 
on two high-volume surgeons, and the validation cohorts consisted of patients treated by both high- and low-volume surgeons. We recently reported that patients who undergo RP performed by high-volume surgeons have a significantly improved outcome relative to those treated by nonhigh-volume surgeons. ${ }^{20}$ Recently, a preoperative nomogram for prostate cancer recurrence after RP was validated on a large cohort of patients managed predominantly in the community setting. ${ }^{21}$ Similar to the present model, the preoperative nomogram exhibited good discrimination ( $c$-index 0.72$)$ but the predictions were overly optimistic when applied to patients treated by community-based urologists (likely some of whom were low-volume surgeons). Thus, when counseling a patient on the basis of the prediction of the nomogram, it may be appropriate to suggest the nomogram overestimates a man's likelihood of success if operated on by a low-volume surgeon.

In developing the nomogram, we have used readily available clinical and pathologic parameters for outcome prediction. Numerous biomarkers that are prognostic for prostate cancer recurrence have been identified by immunohistochemistry in paraffin-embedded tissue, ${ }^{22}$, 23 high throughput gene expression microarrays, ${ }^{24,25}$ and plasma- and serum-based assays. 26,27 However, no biomarker has been shown to improve on or enhance the predictive accuracy of the postoperative nomogram. We recently performed high-throughput gene expression analysis of prostate cancer and developed models that integrated prognostic molecular signatures with the postoperative nomogram. The combined molecular and clinical model had a significant, but modest, improvement over the nomogram alone in cross-validation. ${ }^{25}$ This result is a testament to the robust prognostic information contained within the postoperative nomogram.

In summary, we have enhanced and updated a postoperative nomogram for prostate cancer recurrence after RP by extending the predictions of the model to 10 years and by adjusting the predictions to patients treated contemporaneously. We have avoided specifying the level of risk when adjuvant therapy should be considered as the use of adjuvant therapy is controversial and there is no agreement as to what level of risk would justify its use. We suggest a discussion between the patient and physician regarding the benefits and risk of adjuvant therapy on the basis of accurate estimations of the likelihood of treatment failure.

\section{Acknowledgements}

Supported in part by the National Cancer Institute Grant No. CA92629 SPORE in prostate cancer and by a gift from the Leon Lowenstein Foundation. A.J.S. and F.J.B. are supported in part by grants from the American Foundation for Urologic Disease, a T32 training Grant No. CA-82088 from the National Institutes of Health, and a gift from the Tina and Richard V. Carolan Foundation.

Authors' Disclosures of Potential Conflicts of Interest

Although all authors completed the disclosure declaration, the following author or immediate family members indicated a financial interest. No conflict exists for drugs or devices used in a study if they are not being evaluated as part of the investigation. For a detailed description of the disclosure categories, or for more information about ASCO's conflict of interest policy, please refer to the Author Disclosure Declaration and the Disclosures of Potential Conflicts of Interest section in Information for Contributors.

\begin{tabular}{|c|c|c|c|c|c|c|c|c|}
\hline Authors & Employment & Leadership & Consultant & Stock & Honoraria & Research Funds & Testimony & Other \\
\hline Michael W. Kattan & & Oncovance (A) & & Oncovance (A) & & & & \\
\hline \multicolumn{9}{|c|}{$\begin{array}{ll}\text { Dollar Amount Codes } & \text { (A) }<\$ 10,000\end{array}$} \\
\hline
\end{tabular}

\section{References}

1. Kattan MW, Wheeler TM, Scardino PT. Postoperative nomogram for disease recurrence after radical prostatectomy for prostate cancer. J Clin Oncol 1999;17:1499-1507. [PubMed: 10334537] 
2. Graefen M, Karakiewicz PI, Cagiannos I, et al. Validation study of the accuracy of a postoperative nomogram for recurrence after radical prostatectomy for localized prostate cancer. J Clin Oncol 2002;20:951-956. [PubMed: 11844816]

3. Bianco FJ Jr, Kattan MW, Scardino PT, et al. Radical prostatectomy nomograms in black American men: Accuracy and applicability. J Urol 2003;170:73-77. [PubMed: 12796648]

4. Pound CR, Partin AW, Eisenberger MA, et al. Natural history of progression after PSA elevation following radical prostatectomy. JAMA 1999;281:1591-1597. [PubMed: 10235151]

5. Ward JF, Blute ML, Slezak J, et al. The long-term clinical impact of biochemical recurrence of prostate cancer 5 or more years after radical prostatectomy. J Urol 2003;170:1872-1876. [PubMed: 14532796]

6. Cagiannos I, Karakiewicz P, Graefen M, et al. Is year of radical prostatectomy a predictor of outcome in prostate cancer? J Urol 2004;171:692-696. [PubMed: 14713788]

7. Kaplan EL, Meier P. Nonparametric estimation from incomplete observations. J Am Stat Assoc 1958;53:457-481.

8. Skuladottir H, Olsen JH. Conditional survival of patients with the four major histologic subgroups of lung cancer in Denmark. J Clin Oncol 2003;21:3035-3040. [PubMed: 12915592]

9. Harrell FE Jr, Lee KL, Mark DB. Multivariable prognostic models: Issues in developing models, evaluating assumptions and adequacy, and measuring and reducing errors. Stat Med 1996;15:361387. [PubMed: 8668867]

10. Wheeler TM, Dillioglugil O, Kattan MW, et al. Clinical and pathological significance of the level and extent of capsular invasion in clinical stage T1-2 prostate cancer. Hum Pathol 1998;29:856-862. [PubMed: 9712429]

11. Therneau, TM.; Grambsch, PM. Modeling survival data: Extending the Cox model. New York, NY: Springer Verlag; 2000.

12. Harrell FE Jr, Califf RM, Pryor DB, et al. Evaluating the yield of medical tests. JAMA 1982;247:25432546. [PubMed: 7069920]

13. Cowen ME, Miles BJ, Cahill DF, et al. The danger of applying group-level utilities in decision analyses of the treatment of localized prostate cancer in individual patients. Med Decis Making 1998;18:376-380. [PubMed: 10372579]

14. Blumberg JW. PDA applications for physicians. ASCO News 2004;16:S4-S6.

15. Dillioglugil O, Leibman BD, Kattan MW, et al. Hazard rates for progression after radical prostatectomy for clinically localized prostate cancer. Urology 1997;50:93-99. [PubMed: 9218025]

16. D'Amico AV, Moul JW, Carroll PR, et al. Surrogate end point for prostate cancer-specific mortality after radical prostatectomy or radiation therapy. J Natl Cancer Inst 2003;95:1376-1383. [PubMed: 13130113]

17. Wei JT, Dunn RL, Sandler HM, et al. Comprehensive comparison of health-related quality of life after contemporary therapies for localized prostate cancer. J Clin Oncol 2002;20:557-566. [PubMed: 11786586]

18. Stamey TA, Caldwell M, McNeal JE, et al. The prostate specific antigen era in the United States is over for prostate cancer: What happened in the last 20 years? J Urol 2004;172:1297-1301. [PubMed: 15371827]

19. Bolla M, Van Poppel H, Collette L, et al. Postoperative radiotherapy after radical prostatectomy: A randomized controlled trial (EORTC trial 22911). Lancet 2005;365:572-578. [PubMed: 15708099]

20. Bianco FJ Jr, Scardino PT, Kattan MW, et al. Surgeon volume is a predictor of improved outcomes in radical prostatectomy patients. J Urol 2004;171:211.(abstr 796)

21. Greene KL, Meng MV, Elkin EP, et al. Validation of the Kattan preoperative nomogram for prostate cancer recurrence using a community based cohort: Results from cancer of the prostate strategic urological research endeavor (capsure). J Urol 2004;171:2255-2259. [PubMed: 15126797]

22. Halvorsen OJ, Haukaas SA, Akslen LA. Combined loss of PTEN and p27 expression is associated with tumor cell proliferation by Ki-67 and increased risk of recurrent disease in localized prostate cancer. Clin Cancer Res 2003;9:1474-1479. [PubMed: 12684422]

23. Rhodes DR, Sanda MG, Otte AP, et al. Multiplex biomarker approach for determining risk of prostatespecific antigen-defined recurrence of prostate cancer. J Natl Cancer Inst 2003;95:661-668.

[PubMed: 12734317] 
24. Singh D, Febbo PG, Ross K, et al. Gene expression correlates of clinical prostate cancer behavior. Cancer Cell 2002;1:203-209. [PubMed: 12086878]

25. Stephenson AJ, Smith A, Kattan MW, et al. Integration of gene expression profiling and clinical variables to predict prostate cancer recurrence after radical prostatectomy. Cancer 2005;104:290 298. [PubMed: 15948174]

26. Kattan MW, Shariat SF, Andrews B, et al. The addition of interleukin-6 soluble receptor and transforming growth factor beta1 improves a preoperative nomogram for predicting biochemical progression in patients with clinically localized prostate cancer. J Clin Oncol 2003;21:3573-3579. [PubMed: 12913106]

27. Shariat SF, Kattan MW, Song W, et al. Early postoperative peripheral blood reverse transcription PCR assay for prostate-specific antigen is associated with prostate cancer progression in patients undergoing radical prostatectomy. Cancer Res 2003;63:5874-5878. [PubMed: 14522912] 


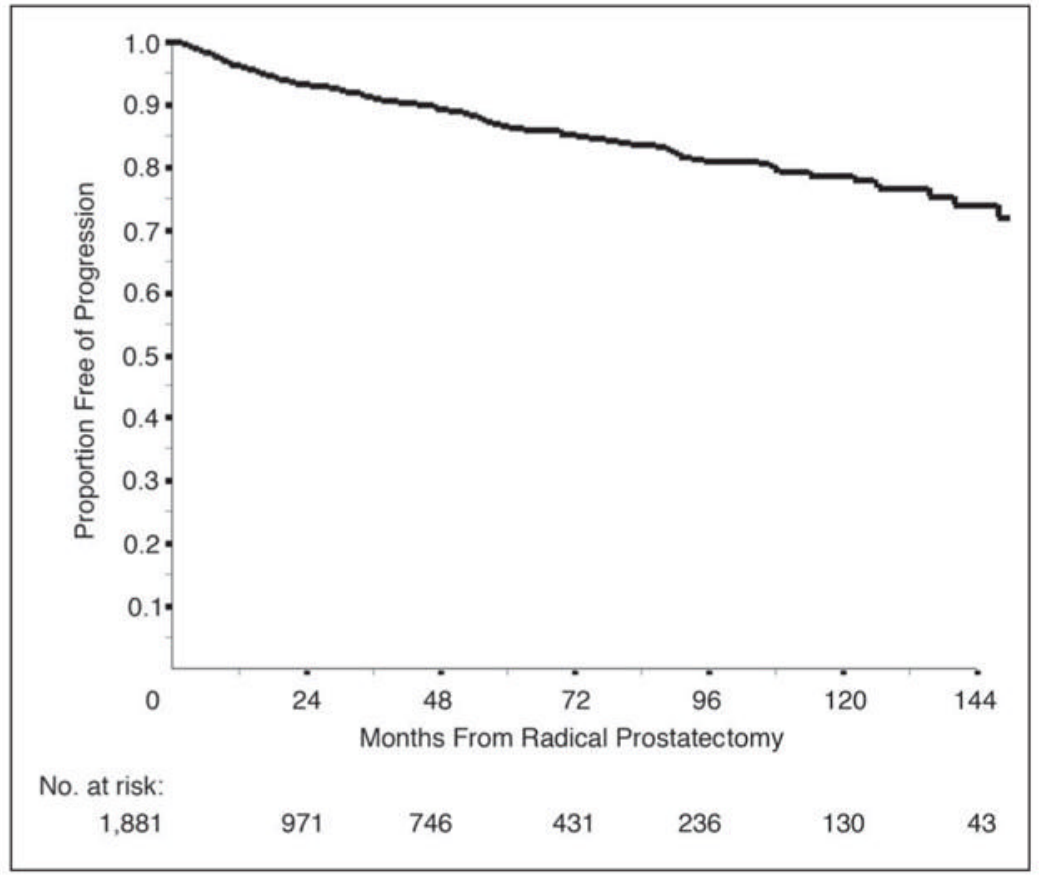

Fig 1.

Overall progression-free probability in the modeling cohort. 


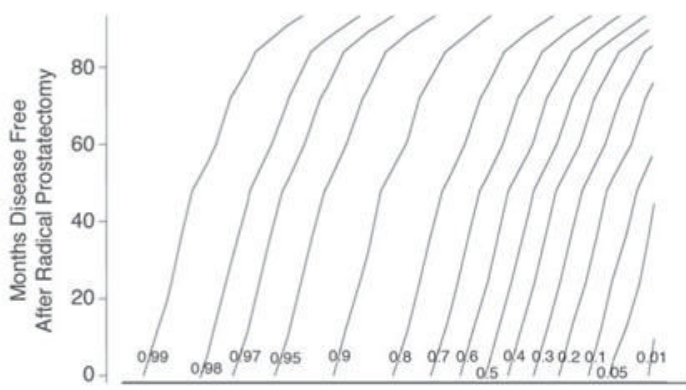

Total Points

$60 \quad 80$

$100 \quad 120$

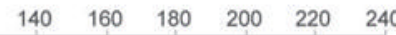

Year of RP

$\begin{array}{cc}2004 & 1996 \\ \text { Pos }\end{array}$

Surgical Margins

Neg

Extracap Extension

\begin{tabular}{lll} 
& & Yes \\
No & & \\
& & \\
& & \\
& Yes & \\
\hline
\end{tabular}

Lymph Node Inv

No

Primary Gleason

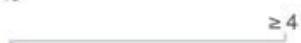

$\leq 3$

34

Secondary Gleason

$\leq 3$

Preoperative PSA

0

0.1

$0.3 \quad 0.7$

$\begin{array}{lllllll}2 & 3 & 5 & 8 & 15 & 40 & 100\end{array}$

Points

$10 \quad 20$
0

30

40

50

Instructions for physician: Locate patient's surgical margin status on the Surgical Margins axis. Draw a straight line down to the Points axis to determine how many points towards disease recurrence that patient receives for his surgical margin status. Repeat this process for each of the remaining axes. Sum the points for each predictor and locate this sum on the Total Points axis. Draw a line straight up from the Total Points axis until it intersects with the horizontal line drawn from the Months Disease-Free After Radical Prostatectomy, corresponding to the number of months the patient has maintained an undetectable PSA (this would equal zero for the immediate postoperative prediction). The slanted vertical line that crosses this intersection point corresponds to the calculated 10-year progression-free probability from the time of radical prostatectomy.

Instruction to patient: "Mr. X, if we had 100 men exactly like you, we would expect <predicted probability from nomogram $\times 100>$ to remain free of biochemical progression at 10 years following radical prostatectomy, and progression after 10 years is exceedingly rare.

Fig 2.

Postoperative nomogram predicting 10-year progression-free probability after radical prostatectomy. RP, radical prostatectomy; extracap, extracapsular; sem ves, seminal vesical; inv, involvement; PSA, prostate-specific antigen. 


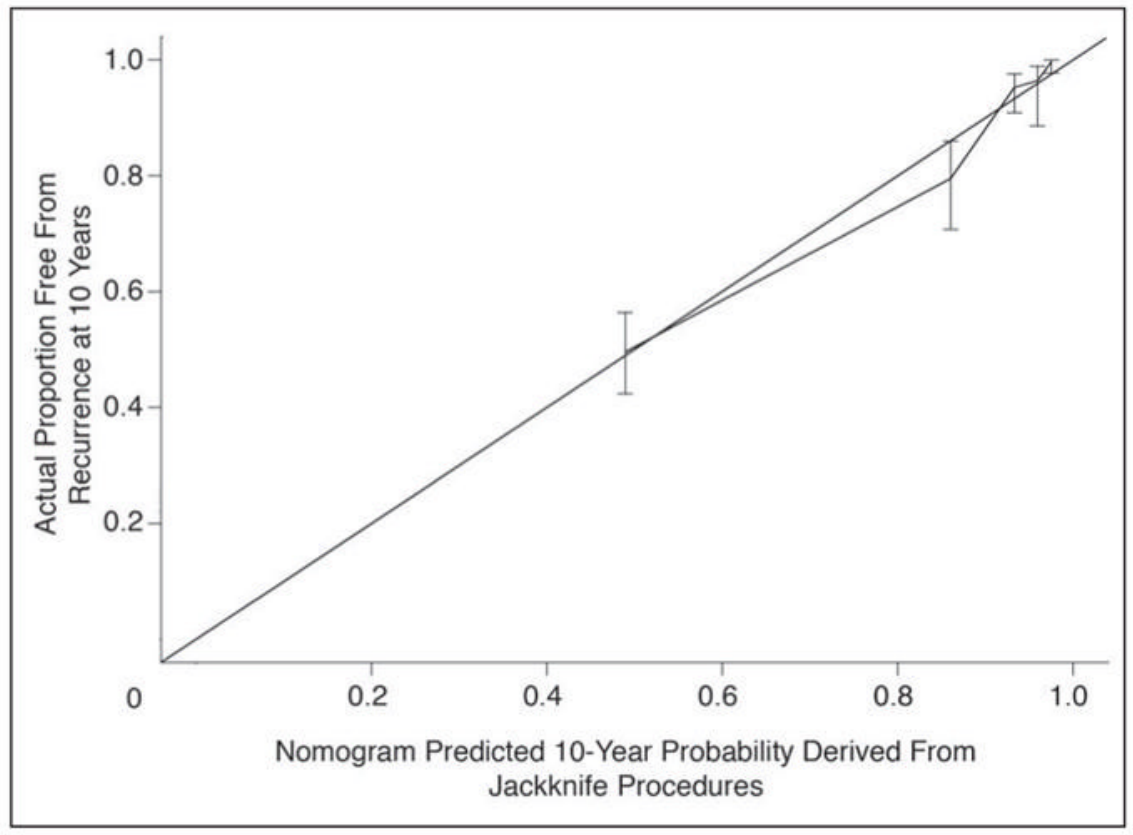

Fig 3.

Calibration of the nomogram based on the jackknife-derived predicted probabilities. 


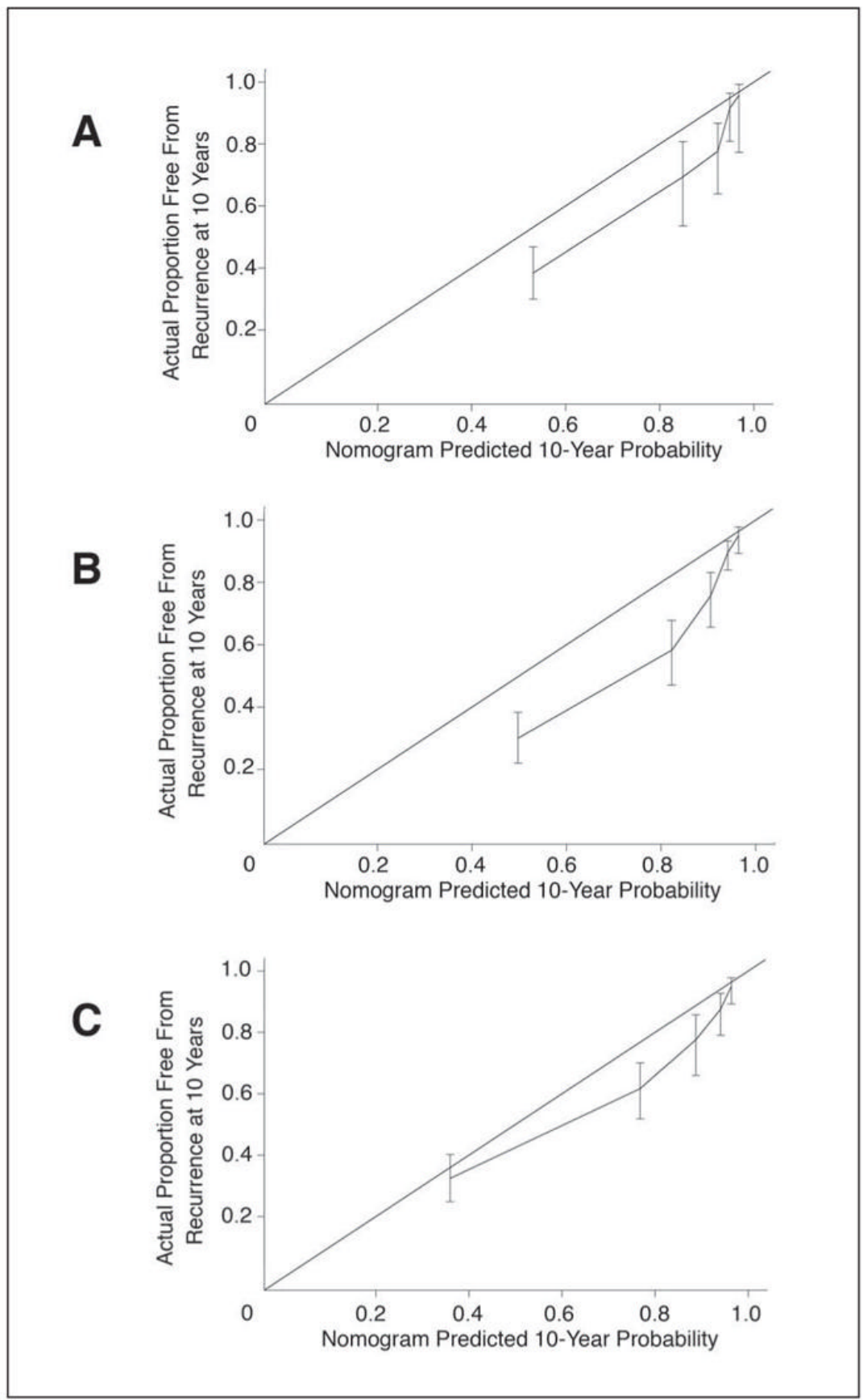

Fig 4.

Calibration of the nomogram when applied to the Memorial Sloan-Kettering Cancer Center validation set and Cleveland Clinic Foundation validation set assuming Gleason 7 patients were Gleason $3+4$ and Gleason $4+3$. 


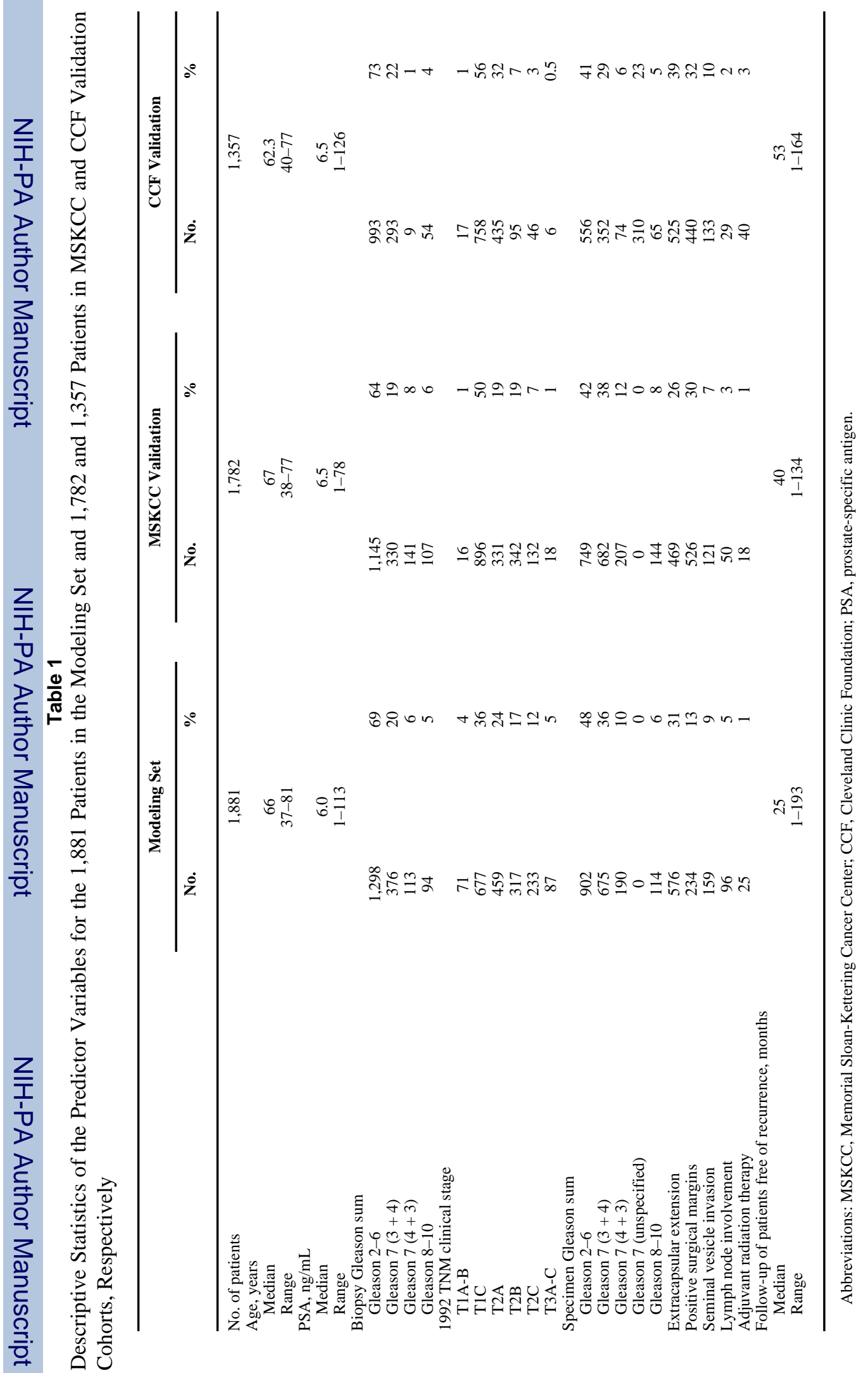

J Clin Oncol. Author manuscript; available in PMC 2008 February 5. 